\title{
LETTERS
}

\section{Supporting the call for a gender-neutral human papillomavirus vaccination in Canada}

We commend Shapiro and colleagues ${ }^{1}$ on their recent policy analysis of human papillomavirus (HPV) vaccination for males in Canada and their call for gender-neutral programs in all provinces and territories.

Australia has the highest uptake of HPV vaccine in males at a country-level worldwide; we write to add evidence that may inform Canada's approach. Quadrivalent HPV vaccine was included for males under the Australian National Immunisation Program from February 2013, following introduction for females in 2007. Australia's nationally centralized systems for economic assessment and vaccine procurement facilitate program decisions: inclusion was deemed cost-effective, albeit at an undisclosed price. $^{2}$

The gender-neutral HPV vaccination program received support even before the female vaccination program commenced. ${ }^{3}$ Uptake in males was high in the first year of the additional program and has increased: $77.0 \%, 74.0 \%$ and $66.4 \%$ of boys turning 15 years of age had received one, two and three doses, respectively, by $2015 .{ }^{4} \mathrm{HPV}$ vaccination coverage for females has also increased by about $6 \%$ since male immunization was funded (from $71.0 \%$ to $77.4 \%$ for three-dose coverage between 2013 and 2015). ${ }^{4}$ Targeting all young people allows more consistent framing of HPV vaccination as a cancer prevention strategy and has likely somewhat negated the vaccine resistance observed in Australia and in other countries. A strong communications strategy, together with strengthening vaccine safety surveillance, ${ }^{5}$ has also facilitated vaccine uptake.

Increasing recognition and incidence of HPV-related diseases like anal and oropharyngeal cancers ${ }^{6}$ and growing evidence of vaccine immunogenicity, safety and efficacy in males from clinical trials ${ }^{7}$ underpins establishment of equitable HPV vaccination programs. Reliance on herd immunity from a female HPV vaccination program to protect males against HPVassociated disease is arguably unethical, particularly in the context of demonstrated cost-effectiveness in high-income countries. We fully support our colleagues in their efforts to advocate for equitable HPV vaccination throughout Canada.

\section{Cyra Patel MBA}

Research Officer, National Centre for Immunisation Research and Surveillance, Westmead NSW, Australia

\section{Kristine Macartney MBBS BMedSci MD}

Deputy Director, National Centre for Immunisation Research and Surveillance, Westmead NSW, Australia, and Associate Professor, University of Sydney, School of Child and Adolescent Health

- Cite as: CMAJ 2017 January 23;189:E118. doi: $10.1503 /$ cmaj.732446

\section{References}

1. Shapiro GK, Perez S, Rosberger Z. Rosberger. Including males in Canadian human papillomavirus vaccination programs: a policy analysis. CMAJ 2016; 188:881-6.

2. Georgousakis M, Jayasinghe S, Brotherton J, et al. Population-wide vaccination against human papillomavirus in adolescent boys: Australia as a case study. Lancet Infect Dis 2012;12:627-34.

3. Marshall H, Ryan P, Roberton D, et al. A crosssectional survey to assess community attitudes to introduction of human papillomavirus vaccine. Aust N Z J Public Health 2007;31:235-42.

4. National HPV Vaccination Program Register. Coverage data. Canberra (AU): Australian Government, Department of Health; 2016. Available: www.hpvregister.org.au/research/ coverage-data (accessed 2016 June 27).

5. Enhanced school-based surveillance of acute adverse events following immunisation with human papillomavirus vaccine in males and females, 2013. Canberra (AU): Australian Government, Department of Health, Therapeutic Goods Administration; 2015.

6. Chaturvedi AK, Anderson WF, Lortet-Tieulent J, et al. Worldwide trends in incidence rates for oral cavity and oropharyngeal cancers. J Clin Oncol 2013;31: 4550-9.

7. Goldstone SE, Jessen H, Palefsky JM, et al. Quadrivalent HPV vaccine efficacy against disease related to vaccine and non-vaccine HPV types in males. Vaccine 2013;31:3849-55. 